\title{
Bootstrapped Newtonian quantum gravity
}

\author{
Roberto Casadio $^{1,2, a}$ (i), Iberê Kuntz ${ }^{1,2, b}$ \\ ${ }^{1}$ Dipartimento di Fisica e Astronomia, Università di Bologna, via Irnerio 46, 40126 Bologna, Italy \\ 2 I.N.F.N., Sezione di Bologna, I.S. FLAG, viale B. Pichat 6/2, 40127 Bologna, Italy
}

Received: 24 March 2020 / Accepted: 15 June 2020 / Published online: 29 June 2020

(C) The Author(s) 2020

\begin{abstract}
We compute quantum corrections for the gravitational potential obtained by including a derivative selfcoupling in its classical dynamics as a toy model for analysing quantum gravity in the strong field regime. In particular, we focus on quantum corrections to the classical solutions in the vacuum outside localised matter sources.
\end{abstract}

\section{Introduction}

In quantum field theory, fundamental forces are associated with the exchange of (virtual) quanta of the interaction fields among matter fields and static potentials only emerge as approximate descriptions for particular configurations. For instance, the linear interaction among fermions in Quantum Electro-Dynamics (QED) is carried by the two polarisations of the massless vector field whose quanta are the photons. The Coulomb potential describing the force acting on a (strictly speaking) static charge then emerges in the nonrelativistic limit of the tree-level transition amplitude for the scattering between two charged fermions via the exchange of these (virtual) photons [1]. For extended sources involving many charged particles, the calculation of amplitudes becomes immediately very cumbersome already at the tree level, and things only get worse when nonlinearities stemming from quantum (loop) corrections are included.

The linear Newtonian interaction likewise emerges from a suitable limit for the exchange of spin 2 (virtual) gravitons between two massive particles in the weak field regime. However, according to General Relativity (GR), nonlinearities should already be present at the classical level, which makes explicit quantum calculations for configurations in the strong field regime very difficult, if possible at all. As an effective description of the gravitational force, say applied on a test particle by an extended matter source, one can

\footnotetext{
a e-mail: casadio@bo.infn.it (corresponding author)

b e-mail: kuntz@bo.infn.it
}

instead consider the static potential as the mean field generated by that extended source and quantise it canonically. This approach applied to QED leads to the description of a static electric field in terms of the coherent state of (virtual) longitudinal photons [2]. It then appears straightforward that one can quantise the Newtonian potential, which solves the classical Poisson equation, in terms of (virtual) scalar gravitons, in a similar fashion. However, for the purpose of studying the strong field regime of gravity, it is more interesting to try and include some nonlinearities [3] in the classical equation for the gravitational potential (the bootstrapped Newtonian gravity introduced in Refs. [4,5]) and then express the resulting solutions in terms of modified coherent states $[6,7]$.

In this work, we will instead quantise the bootstrapped potential as a scalar field in order to determine one-loop quantum corrections to its effective field equation. For this purpose, we will first perform a field redefinition to dispose of the derivative self-interaction and then apply heat kernel techniques in order to compute the quantum effective action [8-13]. Hopefully, this simplified approach to nonlinearities will help to gain some insight about the quantum dynamics of gravity in the strong field regime generated by the presence of matter sources.

This paper is organised as follows: in Sect. 2, we show how we can deal with derivative interactions non-perturbatively by means of a field redefinition that is able to transform the action into a canonical form; in Sect. 3, we review the unique effective action first introduced in Ref. [8] and which allows us to extend the findings of Sect. 2 to the quantum level by requiring the quantum action to be covariant off-shell; Sect. 4 is devoted to the calculation of the quantum action for bootstrapped gravity. We show that the quantum equation of motion is described by a non-local equation in the infrared regime, which is then solved for an idealized pointlike source and for a pair of point-like sources; we finally draw some conclusions in Sect. 5. 


\section{Field redefinition}

We start by recalling that the action for the bootstrapped Newtonian potential $V=V(r)$ for spherically symmetric systems is given by $[4]^{1}$

$$
\begin{aligned}
S[V]= & -4 \pi \int r^{2} \mathrm{~d} r\left[\frac{\left(V^{\prime}\right)^{2}}{8 \pi G_{\mathrm{N}}}\left(1-4 q_{\phi} V\right)\right. \\
& \left.+q_{\mathrm{B}} \rho V\left(1-2 q_{\phi} V\right)\right],
\end{aligned}
$$

where $q_{\phi}$ is a (positive) coupling that controls the potential self-interaction and $q_{\mathrm{B}}$ is introduced to keep track of the coupling with the matter source of density $\rho .^{2}$ We here want to show that the derivative interaction can be transformed into a canonical kinetic term under a field redefinition, which is only possible because the configuration space turns out to have vanishing curvature. This will allow us to quantize the theory non-perturbatively in $q_{\phi}$, although we will still need to invoke perturbation theory in the coupling constant $q_{\mathrm{B}}$ to deal with matter interactions.

We first rescale the (dimensionless) gravitational potential [6]

$\phi=\frac{V}{\sqrt{G_{\mathrm{N}}}}=\sqrt{\frac{m_{\mathrm{p}}}{\ell_{\mathrm{p}}}} V$

the matter density

$J_{\mathrm{B}}=4 \pi \sqrt{G_{\mathrm{N}}} \rho=4 \pi \sqrt{\frac{\ell_{\mathrm{p}}}{m_{\mathrm{p}}}} \rho$,

and promote the new scalar field $\phi=\phi\left(x^{\mu}\right)$ as well as $J_{\mathrm{B}}=$ $J_{\mathrm{B}}\left(x^{\mu}\right)$ to depend on all spacetime coordinates $x^{\mu}=(t, \vec{x})$. The generalised bootstrapped action then reads

$S\left[\phi, J_{B}\right]=S_{0}[\phi]+S_{\mathrm{int}}\left[\phi, J_{B}\right]$,

where

$S_{0}[\phi]=\int \mathrm{d}^{4} x\left[-\frac{1}{2} \partial_{\mu} \phi \partial^{\mu} \phi+\alpha\left(\partial_{\mu} \phi\right)^{2} \phi\right]$

is the kinetic part containing a derivative self-interaction, and

$S_{\mathrm{int}}\left[\phi, J_{B}\right]=\int \mathrm{d}^{4} x \xi(\phi) J_{\mathrm{B}}$

In order to avoid heavy notation, we also introduced

$\alpha=2 q_{\phi} \sqrt{\frac{\ell_{\mathrm{p}}}{m_{\mathrm{p}}}}$

${ }^{1}$ We shall use units with $c=1$, the Newton constant $G_{\mathrm{N}}=\ell_{\mathrm{p}} / m_{\mathrm{p}}$ and $\hbar=\ell_{\mathrm{p}} m_{\mathrm{p}}$.

${ }^{2}$ In the present work we neglect the pressure term analysed in Ref. [5], and just note that it could simply be absorbed into the definition of the matter density. and

$\xi(\phi)=-q_{\mathrm{B}} \phi(1-\alpha \phi)$,

which represents a non-linear coupling to the source $J_{\mathrm{B}}$.

The above action contains derivative interactions, which means that the action for the free field is not recovered by simply setting $q_{\mathrm{B}}=q_{\phi}=0$. But one can perform a field redefinition and try to put it in canonical form by diagonalising the whole kinetic Lagrangian density

$\mathcal{L}_{0}=\left(-\frac{1}{2}+\alpha \phi\right) \partial_{\mu} \phi \partial^{\mu} \phi$.

As can be seen from Eq. (2.9), for $\phi>1 / 2 \alpha$ the kinetic term changes sign and $\phi$ becomes a ghost. For this reason, we will focus on the branch $\phi<1 / 2 \alpha .^{3}$ The dependence on $\phi$ inside the brackets that multiply $\left(\partial_{\mu} \phi\right)^{2}$ also indicates that the metric in field space is not trivially flat, which can either mean that the field space is curved or that the field space is flat but the chosen coordinate is curvilinear. If the field space is flat, then there exists a field transformation which diagonalises the kinetic term. Reciprocally, should there be a frame where the kinetic term is diagonal, then the field space must be flat. We will show below that this is indeed the case for the Lagrangian density (2.9).

To put the kinetic term in the canonical form, we need a field redefinition $\varphi=\psi(\phi)$ such that

$\partial_{\mu} \varphi=\sqrt{1-2 \alpha \phi} \partial_{\mu} \phi$,

which is real and non-singular for

$\phi<\frac{1}{2 \alpha}$,

and is solved by

$\varphi=C-\frac{1}{3 \alpha}(1-2 \alpha \phi)^{3 / 2}$,

where $C$ is an arbitrary integration constant. Upon requiring that the transformation reduces to the identity for $\alpha \rightarrow 0$, we obtain

$\varphi=\psi(\phi)=\frac{1}{3 \alpha}\left[1-(1-2 \alpha \phi)^{3 / 2}\right]$.

Upon inverting the above relation, we get

$\phi=\psi^{-1}(\varphi)=\frac{1}{2 \alpha}\left[1-(1-3 \alpha \varphi)^{2 / 3}\right]$,

which is precisely the relation between the exact "vacuum" solution

$V_{\mathrm{c}}=\frac{1}{4 q_{\phi}}\left[1-\left(1-6 q_{\phi} V_{\mathrm{N}}\right)^{2 / 3}\right]$

\footnotetext{
3 Typical classical solutions are expected to have $\phi<0$, so that this condition is trivially satisfied.
} 
and the Newtonian potential

$V_{\mathrm{N}}=-\frac{G_{\mathrm{N}} M}{r}$,

where $M$ is the source mass. ${ }^{4}$ This is consistent with the fact that the Newtonian potential has canonical kinetic term. In terms of $\varphi$, the complete Lagrangian density then reads

$\mathcal{L}=-\frac{1}{2} \partial_{\mu} \varphi \partial^{\mu} \varphi+\tilde{\xi}(\varphi) J_{\mathrm{B}}$,

where the non-linear coupling $\tilde{\xi}(\varphi)=\xi\left(\psi^{-1}(\varphi)\right)$ is given by

$\tilde{\xi}(\varphi)=-\frac{q_{\mathrm{B}}}{4 \alpha}\left[1-(1-3 \alpha \varphi)^{4 / 3}\right]$.

Since the interaction terms do not contain any derivatives, the Lagrangian density (2.17) for $\varphi$ can be quantised in the standard way by defining the asymptotic states for the free field $\varphi_{0}$.

\section{Covariant quantum action}

The quantisation of the theory will be performed using the one particle irreducible (1PI, or quantum) action. The calculation of the 1PI action in our case is a little subtler than the standard calculations in quantum field theory because of the curvilinear coordinates in the original frame (2.4). In particular, the naive definition of the 1PI action is frame dependent off-shell and can only be used if one is interested in the components of the S-matrix, which are calculated on-shell. On the other hand, we are interested in the dynamics of the off-shell mean field in a given state, whose quantum action would produce non-covariant results. Fortunately, a covariant formulation of the 1PI action exists as it was introduced in Ref. [8] (see also [9]). In the following, we briefly review such formulation in one-dimensional field space, which is enough for our purposes since the action (2.4) contains only one degree of freedom.

The covariant partition function is given by

$Z[J]=\int \mathcal{D} \phi e^{i\{S[\phi]+[v[\bar{\phi}]-\sigma(\bar{\phi}, \phi)] J\}}$,

where $v[\bar{\phi}]$ is an arbitrary covector field satisfying $\nabla_{\bar{\phi}} v[\bar{\phi}]=$ 1 with $\nabla_{\bar{\phi}}$ being the covariant derivative associated with the Levi-Civita connection $\gamma(\bar{\phi})$ of the field space, the displacement vector is defined by $\sigma(\bar{\phi}, \phi)=\partial^{\bar{\phi}} \tilde{\sigma}(\bar{\phi}, \phi)$ and $\tilde{\sigma}(\bar{\phi}, \phi)$ is Synge's world function, which is numerically equal to onehalf of the square of the geodesic distance between $\phi$ and $\bar{\phi}$. Because $v[\bar{\phi}]$ transforms as a covector and $\sigma(\bar{\phi}, \phi)$ transforms as a covector with respect to its first argument and as a

\footnotetext{
$\overline{4}$ We shall see later on that the mass $M$ however differs from the Newtonian expectation [4].
}

scalar with respect to its second argument, the partition function $Z[J]$ is completely covariant under redefinitions of the background and of the quantum fields. The covariant relation between the background field and the mean field is now given by

$$
\langle\sigma(\bar{\phi}, \phi)\rangle=0 \text {, }
$$

instead of the naive relation $\bar{\phi}=\langle\phi\rangle$. The displacement vector can be expanded as

$$
-\sigma(\bar{\phi}, \phi)=\phi-\bar{\phi}+\frac{1}{2} \gamma(\bar{\phi})(\phi-\bar{\phi})^{2}+\mathcal{O}(\phi-\bar{\phi})^{3},
$$

where $\gamma(\bar{\phi})$ is the one-dimensional Levi-Civita connection in field space, thus one obviously retrieve the naive definition $\bar{\phi}=\langle\phi\rangle$ for a vanishing connection. The covariant 1PI action is defined as

$\Gamma[\bar{\phi}]=W[J]-v[\bar{\phi}] J, \quad$ with $\frac{\delta W[J]}{\delta J}=v[\bar{\phi}]$,

where $W[J]=-i \log Z[J]$ is the generating functional of connected diagrams. From Eqs. (3.1) and (3.4), one obtains

$e^{i \Gamma[\bar{\phi}]}=\int \mathcal{D} \phi e^{i\left\{S[\phi]+\sigma(\bar{\phi}, \phi) \nabla_{\bar{\phi}} \Gamma[\bar{\phi}]\right\}}$.

To perform the integral in Eq. (3.5), it is best to make the change of variables

$\phi \rightarrow \sigma(\bar{\phi}, \phi)$.

By expanding the classical action as a covariant Taylor series

$S[\phi]=\sum_{n=0}^{\infty} \frac{(-1)^{n}}{n !}\left\{\nabla_{\sigma} S[\bar{\phi}] \sigma(\bar{\phi}, \phi)\right\}^{n}$

and the quantum action as a loop expansion

$\Gamma[\bar{\phi}]=S[\bar{\phi}]+\hbar \Gamma^{(1)}[\bar{\phi}]+\hbar^{2} \Gamma^{(2)}[\bar{\phi}]+\ldots$,

one can calculate the quantum action order by order in $\hbar$. At one-loop order, we find

$\Gamma[\bar{\phi}]=S[\bar{\phi}]+\frac{i \hbar}{2} \operatorname{Tr} \log \left(\nabla^{2} S[\bar{\phi}]\right)$,

where $\operatorname{Tr}$ denotes the functional trace. The only difference with respect to the standard quantum action is the appearance of the functional covariant derivative instead of the standard functional derivative, thus making $\Gamma[\bar{\phi}]$ a covariant object under field redefinitions.

\section{Quantum action for bootstrapped gravity}

The result in the previous section could be applied to the Lagrangian density (2.17) by interpreting $J=J_{\mathrm{B}}$ as the auxiliary source. An alternative procedure would rather be 
to introduce a different auxiliary source $J,{ }^{5}$ in which case the partition function would be given by

$Z\left[J, J_{B}\right]=\int \mathcal{D} \phi e^{i\left\{S\left[\phi, J_{B}\right]+[v[\bar{\phi}]-\sigma(\bar{\phi}, \phi)] J\right\}}$.

In this way, $J$ can be simply interpreted like a Lagrange multiplier, which will be set to zero in the end as usual, and needs not necessarily be a physical source. The 1PI action results in

$$
\begin{aligned}
e^{i \Gamma\left[\bar{\phi}, J_{\mathrm{B}}\right]} & =\int \mathcal{D} \phi e^{i\left\{S\left[\phi, J_{\mathrm{B}}\right]+\sigma(\bar{\phi}, \phi) \nabla_{\bar{\phi}} \Gamma\left[\bar{\phi}, J_{\mathrm{B}}\right]\right\}} \\
& =\int \mathcal{D} \varphi e^{i\left\{\tilde{S}\left[\varphi, J_{\mathrm{B}}\right]+\sigma\left(\psi^{-1}(\bar{\varphi}), \psi^{-1}(\varphi)\right) \nabla_{\bar{\phi}} \tilde{\Gamma}\left[\bar{\varphi}, J_{\mathrm{B}}\right]\right\}},
\end{aligned}
$$

where in the second line we used the redefinition (2.14) and denoted

$$
\begin{aligned}
\tilde{S}\left[\varphi, J_{\mathrm{B}}\right] & =S\left[\psi^{-1}(\varphi), J_{\mathrm{B}}\right] \\
& =\int \mathrm{d}^{4} x\left[-\frac{1}{2} \partial_{\mu} \varphi \partial^{\mu} \varphi+\tilde{\xi}(\varphi) J_{\mathrm{B}}\right],
\end{aligned}
$$

and $\tilde{\Gamma}\left[\varphi, J_{\mathrm{B}}\right]=\Gamma\left[\psi^{-1}(\varphi), J_{\mathrm{B}}\right]$. As it was already pointed out in Sect. 2, in the new frame the field coordinates are Cartesian. The covariant functional derivative thus reduces to the flat form $\nabla_{\varphi}=\frac{\delta}{\delta \varphi}$, and the displacement vector reduces to the coordinate difference in field space

$\sigma\left(\psi^{-1}(\bar{\varphi}), \psi^{-1}(\varphi)\right)=\psi^{-1}(\bar{\varphi})-\psi^{-1}(\varphi)$.

The quantum action to one-loop order then reads

$\tilde{\Gamma}\left[\bar{\varphi}, J_{\mathrm{B}}\right]=\tilde{S}\left[\bar{\varphi}, J_{\mathrm{B}}\right]+\frac{i \hbar}{2} \operatorname{Tr} \log \Delta$,

with

$$
\begin{aligned}
\Delta & =\square+\tilde{\xi}^{\prime \prime}(\bar{\varphi}) J_{\mathrm{B}} \\
& =\square+q_{\mathrm{B}} \alpha(1-3 \alpha \bar{\varphi})^{-2 / 3} J_{\mathrm{B}},
\end{aligned}
$$

where $\square=\eta^{\mu \nu} \partial_{\mu} \partial_{\nu}$ denotes the D'Alembert operator in flat spacetime. To calculate the second term in Eq. (4.6), we use the Schwinger proper time method to represent the one-loop functional determinant in terms of the heat kernel $K$ of the operator $\Delta$ as

$\operatorname{Tr} \log \Delta=-\int_{0}^{\infty} \mathrm{d} s \frac{\operatorname{Tr} K(s)}{s}$.

In the presence of a potential term in $\Delta$, such as $P \equiv$ $\tilde{\xi}^{\prime \prime}(\bar{\varphi}) J_{\mathrm{B}}$ in Eq. (4.7), the computation of the exact heat kernel becomes highly non-trivial and it is necessary to

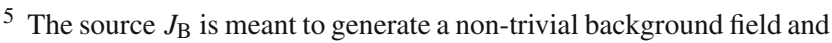
cannot be made to vanish arbitrarily.
}

rely on approximate methods. Different approximation techniques with different scopes of applicability have been developed [10-13], the most popular one perhaps being the Schwinger-DeWitt expansion in inverse powers of the field mass. However, the Schwinger-DeWitt expansion obviously breaks down for massless theories, like our action (2.4), only being able to produce the divergent part of the quantum action.

A useful tool to study heat kernels in the absence of a mass term is the covariant perturbation theory $[12,13]$, which is based on an asymptotic expansion in terms of spacetime curvatures, fibre bundle curvatures (gauge field strengths) and potential terms and can be seen as a resummation of the Schwinger-DeWitt expansion. In our case, there are no curvatures present, either in spacetime or in field space, and the trace of the heat kernel can only be expanded as a series in the potential term $P$ as

$$
\begin{aligned}
\operatorname{Tr} K(s)= & \frac{1}{(4 \pi s)^{\omega}} \int \mathrm{d}^{4} x[1+s P \\
& \left.+s^{2} P f(-s \square) P+\mathcal{O}\left(P^{3}\right)\right],
\end{aligned}
$$

where

$f(u)=\frac{1}{2} \int_{0}^{1} \mathrm{~d} t e^{-t(1-t) u}$.

Substituting (4.9) in Eq. (4.8) and changing the order of integration, we find the one-loop contribution to the 1PI action to second order in the potential term $P$ is given by

$$
\begin{gathered}
\tilde{\Gamma}^{(1)}[\bar{\varphi}]=\frac{q_{\mathrm{B}}^{2} \alpha^{2}}{4(4 \pi)^{\omega}} \int \mathrm{d}^{4} x \frac{J_{\mathrm{B}}}{(1-3 \alpha \bar{\varphi})^{2 / 3}}\left[\frac{1}{2-\omega}+2\right. \\
\left.-\log \left(\frac{-\square}{\mu^{2}}\right)\right] \frac{J_{\mathrm{B}}}{(1-3 \alpha \bar{\varphi})^{2 / 3}}
\end{gathered}
$$

for $\omega \rightarrow 2$. Here $\mu$ is a normalisation mass necessary to make the logarithm dimensionless and whose arbitrariness reflects the renormalisation arbitrariness of the oneloop effective action. Note that the divergence is proportional to $(1-3 \alpha \bar{\varphi})^{-4 / 3}$, whereas the bare action only contains $(1-3 \alpha \bar{\varphi})^{4 / 3}$, which indicates that the theory is nonrenormalisable and only makes sense as an effective field theory. Since the only mass parameter present in the theory is the Planck mass $m_{\mathrm{p}}$, one indeed expects that the theory breaks down at energies of the order of $m_{\mathrm{p}}$.

Albeit being non-renormalisable, the quantum action can and must be renormalised order by order in the loop expansion in order to produce sensible results. This is done by regarding $\tilde{S}\left[\bar{\varphi}, J_{\mathrm{B}}\right]$ as the bare action and adding counterterms in an expansion in powers of $\hbar$, to wit

$$
\tilde{S}\left[\bar{\varphi}, J_{\mathrm{B}}\right]=\tilde{S}^{(0)}\left[\bar{\varphi}, J_{\mathrm{B}}\right]+\hbar \tilde{S}^{(1)}\left[\bar{\varphi}, J_{\mathrm{B}}\right]+\mathcal{O}\left(\hbar^{2}\right) .
$$


The ultraviolet divergences in Eq. (4.11) can then be eliminated with the choice

$$
\begin{aligned}
& \tilde{S}^{(1)}\left[\bar{\varphi}, J_{\mathrm{B}}\right] \\
& =\tilde{S}_{\mathrm{R}}^{(1)}\left[\bar{\varphi}, J_{\mathrm{B}}\right]-\frac{q_{\mathrm{B}}^{2} \alpha^{2}}{4(4 \pi)^{\omega}} \int \mathrm{d}^{4} x \frac{J_{\mathrm{B}}}{(1-3 \alpha \bar{\varphi})^{2 / 3}} \\
& \quad \times\left(\frac{1}{2-\omega}+2\right) \frac{J_{\mathrm{B}}}{(1-3 \alpha \bar{\varphi})^{2 / 3}},
\end{aligned}
$$

where $\tilde{S}_{\mathrm{R}}^{(1)}$ denotes the action with renormalised coupling constants. Note that we included in $\tilde{S}^{(1)}$ a non-divergent term, which is local and does not contribute to the infrared physics. Thus we can eliminate it via a finite renormalisation for convenience. After renormalisation, the infrared equation of motion is finally given by

$$
\begin{aligned}
& \square \bar{\varphi}=q_{\mathrm{B}} J_{\mathrm{B}}(1-3 \alpha \bar{\varphi})^{1 / 3} \\
& +\left.\frac{q_{\mathrm{B}}^{2} \alpha^{3} \hbar}{16 \pi^{2}} \frac{J_{\mathrm{B}}}{(1-3 \alpha \bar{\varphi})^{5 / 3}} \log \left(-\frac{\square}{\mu^{2}}\right)\right|_{R} \frac{J_{\mathrm{B}}}{(1-3 \alpha \bar{\varphi})^{2 / 3}} .
\end{aligned}
$$

The subscript $R$ in the log operator is a reminder that we must impose retarded boundary conditions by replacing the Feynman Green's function with the retarded one. This procedure, albeit seemingly ad-hoc, results from the in-in path integral formalism and it is required in order to obtain a causal evolution for the mean field $[12,14-16]$. Since the field coordinates in the new frame are Cartesian, we have $\psi^{-1}(\bar{\varphi})=\left\langle\psi^{-1}(\varphi)\right\rangle$. Therefore, solutions to Eq. (4.14) will correspond to corrections to the Newtonian potential in the vacuum state.

We should note that we followed a bottom-up approach to quantum gravity, starting from a modified Newtonian theory of gravity, by promoting the Newtonian potential to a scalar field and then quantising it. Nothing in this construction suggests that the resulting theory exhibits any gauge invariance, thus we need not worry about the usual complications that arise in gauge theories. However, from a top-down viewpoint, gauge symmetry is required to account for the background independence of gravity. The reason why gauge symmetry is not realised in bootstrapped gravity is that we phrased the entire approach in terms of the bootstrapped potential, which is observable ${ }^{6}$ and therefore gauge-invariant. Should one try to formulate the theory in terms of non-observable quantities, gauge fixing conditions are expected to become necessary at the quantum level. A complete analysis of gauge-invariance requires a reconstruction of the full spacetime metric, which is however left for future investigations.

Since it does not look possible to solve exactly Eq. (4.14), we will expand the solution in powers of the coupling $q_{\mathrm{B}}$ as

$\bar{\varphi}=q_{\mathrm{B}} \bar{\varphi}^{(1)}+q_{\mathrm{B}}^{2} \bar{\varphi}^{(2)}+\ldots$,

\footnotetext{
${ }^{6}$ For instance, the potential determines the radial acceleration of a static particle, which is directly observable.
}

and solve Eq. (4.14) perturbatively up to second order in $q_{\mathrm{B}}$. For the static potential $\bar{\varphi}=\bar{\varphi}(\vec{x})$ generated by a static source $J_{\mathrm{B}}=J_{\mathrm{B}}(\vec{x})$, we find

$\nabla^{2} \bar{\varphi}^{(1)}=J_{\mathrm{B}}$

$\nabla^{2} \bar{\varphi}^{(2)}=-\alpha J_{\mathrm{B}} \varphi^{(1)}+\frac{\alpha^{3} \hbar}{16 \pi^{2}} J_{\mathrm{B}} \log \left(-\frac{\nabla^{2}}{\mu^{2}}\right) J_{\mathrm{B}}$,

whose solution is

$$
\begin{aligned}
\bar{\varphi}^{(1)}(\vec{x})= & \int \mathrm{d}^{3} x^{\prime} G\left(\vec{x}, \vec{x}^{\prime}\right) J_{\mathrm{B}}\left(\vec{x}^{\prime}\right), \\
\bar{\varphi}^{(2)}(\vec{x})= & -\alpha \int \mathrm{d}^{3} x^{\prime} G\left(\vec{x}, \vec{x}^{\prime}\right) J_{\mathrm{B}}\left(\vec{x}^{\prime}\right) \bar{\varphi}^{(1)}\left(\vec{x}^{\prime}\right) \\
& +\frac{\alpha^{3} \hbar}{16 \pi^{2}} \int \mathrm{d}^{3} x^{\prime} \int \mathrm{d}^{3} x^{\prime \prime} G\left(\vec{x}, \vec{x}^{\prime}\right) J_{\mathrm{B}}\left(\vec{x}^{\prime}\right) \\
& L\left(\vec{x}^{\prime}, \vec{x}^{\prime \prime}\right) J_{\mathrm{B}}\left(\vec{x}^{\prime \prime}\right),
\end{aligned}
$$

where

$G\left(\vec{x}, \vec{x}^{\prime}\right)=-\frac{1}{4 \pi\left|\vec{x}-\vec{x}^{\prime}\right|}$

is the Green function for the Laplace operator $\nabla^{2}$. Moreover, the kernel $L$ of the log operator,

$\log \left(-\frac{\nabla^{2}}{\mu^{2}}\right) f(x)=\int \mathrm{d}^{3} x^{\prime} L\left(\vec{x}, \vec{x}^{\prime}\right) f\left(\vec{x}^{\prime}\right), \quad \forall f(x)$,

is defined as a pseudo-differential operator acting via the Fourier transform,

$$
\begin{aligned}
L\left(\vec{x}, \vec{x}^{\prime}\right) & =\int \frac{\mathrm{d}^{3} q}{(2 \pi)^{3}} e^{-i \vec{q} \cdot\left(\vec{x}-\vec{x}^{\prime}\right)} \log \left(\frac{q^{2}}{\mu^{2}}\right) \\
& =-\frac{1}{2 \pi\left|\vec{x}-\vec{x}^{\prime}\right|^{3}} .
\end{aligned}
$$

Let us now look at some examples.

\subsection{A point-like source}

For the case of a point-like source of mass $M_{0}$ and current $^{7}$

$J_{\mathrm{B}}(x)=4 \pi \sqrt{G_{\mathrm{N}}} M_{0} \delta(\vec{x})$,

we obtain the standard Newtonian potential (2.16) with $M=$ $M_{0}$ from Eq. (4.18) by defining $r \equiv|\vec{x}|$ and setting $q_{\mathrm{B}}=1$,

$\bar{V}_{\mathrm{N}}=\sqrt{G_{\mathrm{N}}} \bar{\varphi}^{(1)}=-\frac{G_{\mathrm{N}} M_{0}}{r}$.

The correction at order $q_{\mathrm{B}}^{2}$ is likewise obtained from Eq. (4.19) and reads

$\bar{\varphi}^{(2)}=-\lim _{\epsilon \rightarrow 0} \frac{\alpha G_{\mathrm{N}} M_{0}^{2}}{r \epsilon}\left(1-\frac{\alpha^{2} \hbar}{8 \pi^{2}} \frac{1}{\epsilon^{2}}\right)$,

\footnotetext{
$\overline{7}$ Notice that we are taking into account the rescalings (2.2) and (2.3).
} 
which diverges due to the ultra-localized source (4.23). One instead expects finite results when the Dirac delta is replaced by an extended source (whose radius is greater than the Planck length). This is physically expected because strong quantum gravitational effects become important when one probes Planckian distances and they cannot be accounted for within the realm of effective field theory. Nonetheless, these divergences can be removed by any regularisation method or, formally, by choosing an integration contour which does not enclose the spatial origin. With that in mind, we will drop them out and focus on finite terms, which therefore leads to vanishing corrections for the Newtonian potential (4.24). By mapping $\varphi$ back to the original field $\phi$ according to Eq. (2.14), one then precisely recovers the classical solution $V_{\text {c }}$ in Eq. (2.15) with $M=M_{0}$.

We will show in our next example that the above divergences indeed reflect short-distance effects taking place at Planckian scales by considering two point-like sources. This way, an extended body can be simulated, with the distance between the sources determining the body's size. We will also recover the result that the mass $M$ in the bootstrapped potential is not equal to the proper mass $M_{0}$ of the source.

\subsection{Two point-like sources}

We will next solve Eqs. (4.18) and (4.19) for two massive point-like sources of equal mass $M_{0} / 2$ located at points of coordinate $\vec{x}_{1}$ and $\vec{x}_{2}$ and contributing to the total source as

$J_{\mathrm{B}}(x)=2 \pi \sqrt{G_{\mathrm{N}}} M_{0}\left[\delta\left(\vec{x}-\vec{x}_{1}\right)+\delta\left(\vec{x}-\vec{x}_{2}\right)\right]$.

Eq. (4.18) gives again the classical Newtonian potential

$\sqrt{G_{\mathrm{N}}} \bar{\varphi}^{(1)}=-\frac{G_{\mathrm{N}} M_{0}}{2}\left(\frac{1}{\left|\vec{x}-\vec{x}_{1}\right|}+\frac{1}{\left|\vec{x}-\vec{x}_{2}\right|}\right)$.

If we assume that $\left|\vec{x}_{1}-\vec{x}_{2}\right| \equiv R \ll\left|\vec{x}-\vec{x}_{1}\right| \simeq\left|\vec{x}-\vec{x}_{2}\right| \equiv r$, we can in fact write

$\sqrt{G_{\mathrm{N}}} \bar{\varphi}^{(1)} \simeq-\frac{G_{\mathrm{N}} M_{0}}{r}$,

which reproduces the result in the previous example to first order in $q_{\mathrm{B}}$.

For the calculation of $\bar{\varphi}^{(2)}$, we must point out that the product of quantities evaluated at the same point $\vec{x}_{i}$, such as $\delta\left(\vec{x}-\vec{x}_{i}\right) \bar{\varphi}^{(1)}\left(\vec{x}_{i}\right)$, is ill-defined. This is analogous to the situation of the previous example and only reflects the existence of short-distance effects beyond the grasp of the effective field theory. We will therefore drop single-point quantities and focus on the cross terms of quantities evaluated at $\vec{x}_{1}$ and $\vec{x}_{2}$. Eq. (4.19) then gives

$$
\begin{aligned}
\bar{\varphi}^{(2)}= & -\frac{\alpha G_{\mathrm{N}} M_{0}^{2}}{2\left|\vec{x}_{1}-\vec{x}_{2}\right|}\left(1-\frac{\alpha^{2} \hbar}{8 \pi^{2}\left|\vec{x}_{1}-\vec{x}_{2}\right|^{2}}\right) \\
& \times\left(\frac{1}{\left|\vec{x}-\vec{x}_{1}\right|}+\frac{1}{\left|\vec{x}-\vec{x}_{2}\right|}\right) .
\end{aligned}
$$

Finally, the total solution to order $q_{\mathrm{B}}^{2}$ reads

$$
\begin{aligned}
& \sqrt{G_{\mathrm{N}}} \bar{\varphi} \\
& \simeq-\frac{q_{\mathrm{B}} G_{\mathrm{N}} M_{0}}{2}\left[1+\frac{q_{\mathrm{B}} q_{\phi} G_{\mathrm{N}} M_{0}}{\left|\vec{x}_{1}-\vec{x}_{2}\right|}\left(1-\frac{q_{\phi}^{2} \ell_{\mathrm{p}} \hbar}{2 \pi^{2} m_{\mathrm{p}}\left|\vec{x}_{1}-\vec{x}_{2}\right|^{2}}\right)\right] \\
&\left(\frac{1}{\left|\vec{x}-\vec{x}_{1}\right|}+\frac{1}{\left|\vec{x}-\vec{x}_{2}\right|}\right) \\
& \simeq-\frac{G_{\mathrm{N}} M_{0}}{r}\left[1+\frac{q_{\phi} G_{\mathrm{N}} M_{0}}{\left|\vec{x}_{1}-\vec{x}_{2}\right|}\left(1-\frac{q_{\phi}^{2} \ell_{\mathrm{p}}^{2}}{2 \pi^{2}\left|\vec{x}_{1}-\vec{x}_{2}\right|^{2}}\right)\right],
\end{aligned}
$$

in which we used Eq. (2.7) to display the original coupling $q_{\phi}$ and again set $q_{\mathrm{B}}=1$ at the end. Note that one recovers the classical Newtonian potential either for $q_{\phi}=\alpha=0$ or, more formally, when the sources are far away from each other, $\left|\vec{x}_{1}-\vec{x}_{2}\right| \rightarrow \infty$. At first order in $q_{\phi} \sim \alpha$ we have the classical contribution to the non-linearity introduced in the action (2.5), whereas at third order we find the correction due to (one-loop) quantum gravity. We are also able to interpret the divergences in Eq. (4.25) for a single point-like particle as indeed originated from the limit $\vec{x}_{1} \rightarrow \vec{x}_{2}$ in Eq. (4.30). Although we still had to deal with the single-point divergences mentioned above, they will presumably yield finite results once a smooth matter source comprising of all macroscopical sources, is used as opposed to a system of point-like sources.

Note that the above expression (4.30) more accurately reproduces the classical solution $V_{\mathrm{c}}$ in Eq. (2.15) outside an extended source of small compactness $G_{\mathrm{N}} M_{0} \ll R$. In fact, we can consider the distance between the two point sources as the size $R$ of an extended source, that is $R \simeq\left|\vec{x}_{1}-\vec{x}_{2}\right|$, and introduce the modified mass

$$
\begin{aligned}
\tilde{M} & \equiv M_{0}\left[1+q_{\phi} \frac{G_{\mathrm{N}} M_{0}}{R}\left(1-\frac{q_{\phi}^{2} \ell_{\mathrm{p}}^{2}}{2 \pi^{2} R^{2}}\right)\right] \\
& \simeq M\left(1-\frac{q_{\phi}^{2} \ell_{\mathrm{p}}^{2}}{2 \pi^{2} R^{2}}\right)
\end{aligned}
$$

where the relation between $M$ and $M_{0}$ is the same as the one found in Ref. [4] for a uniform star of small compactness, modulo a numerical coefficient of order one. We then obtain

$\sqrt{G_{\mathrm{N}}} \bar{\varphi} \simeq-\frac{G_{\mathrm{N}} \tilde{M}}{r}$.

By transforming back to the original frame $\sqrt{G_{\mathrm{N}}} \phi=V$, we then recover the classical bootstrapped potential (2.15) in the vacuum with the mass $M$ now further corrected by a one-loop contribution. ${ }^{8}$ We remark that the expression for the mass (4.31) only holds for small compactness of the source

\footnotetext{
8 We note in passing that this contribution is consistently of the same order $\ell_{\mathrm{p}}^{2}$ as the corrections found in Ref. [17] for the metric generated by a star.
} 
(that is for $G_{\mathrm{N}} M_{0} / R \ll 1$ ) and that $M>M_{0}$ is precisely a consequence of the nonlinearity included in the bootstrapped dynamics, as discussed in Refs. [4,5].

Finally, we must emphasize that the above solution is nonperturbative in $q_{\phi}$ and should reproduce all effects due to the non-linear self-coupling of gravity. On the other hand, the coupling to matter was handled perturbatively. Treating both $q_{\phi}$ and $q_{\text {B }}$ non-perturbatively is utterly difficult, but one can study non-perturbative effects due to the gravitational selfcoupling at the expense of dealing perturbatively with respect to the coupling to matter.

\section{Conclusions}

In this paper, we have considered non-linear derivative selfinteractions of the Newtonian potential by allowing the first few post-Newtonian terms to take arbitrary values. Such a theory has been called bootstrapped Newtonian gravity. We calculated one-loop quantum corrections to the bootstrapped potential by first promoting the non-relativistic potential to a Lorentz covariant form that allows the application of quantum field theory techniques in intermediate steps. These intermediate calculations are obviously supposed to serve only as a guideline for the quantisation of the complete theory of gravity (e.g. light bending requires a non-scalar gravitational field), thus we must take the non-relativistic limit in the end, which is all we need for our purposes.

We showed that the bootstrapped Newtonian potential is described by a non-local equation of motion in the infrared, which is typical of massless theories. We solved it for a pointlike source and a system of two point-like sources. The latter can be thought of as a rough approximation of an extended source. Our results recover Newtonian physics in the limit where the sources are far apart and for vanishing derivative interactions $\alpha \rightarrow 0$, as one would expect. The analysis of more realistic situations, such as a smooth extended source, proves much more challenging already at the classical level [5] and will be left for future investigations. In any case, the effective equations of motion together with the resulting quantum bootstrapped potential permits a better understanding of quantum processes taking place at non-perturbative settings which are important for strong field applications.
Acknowledgements R.C. and I.K. are partially supported by the INFN Grant FLAG. The work of R.C. has also been carried out in the framework of activities of the National Group of Mathematical Physics (GNFM, INdAM) and COST action Cantata.

Data Availability Statement This manuscript has no associated data or the data will not be deposited. [Authors' comment: This is a theoretical study and no experimental data has been listed.]

Open Access This article is licensed under a Creative Commons Attribution 4.0 International License, which permits use, sharing, adaptation, distribution and reproduction in any medium or format, as long as you give appropriate credit to the original author(s) and the source, provide a link to the Creative Commons licence, and indicate if changes were made. The images or other third party material in this article are included in the article's Creative Commons licence, unless indicated otherwise in a credit line to the material. If material is not included in the article's Creative Commons licence and your intended use is not permitted by statutory regulation or exceeds the permitted use, you will need to obtain permission directly from the copyright holder. To view a copy of this licence, visit http://creativecomm ons.org/licenses/by/4.0/.

Funded by $\mathrm{SCOAP}^{3}$.

\section{References}

1. C. Itzykson, J.B. Zuber, Quantum Field Theory (McGraw-Hill, New York, 1980)

2. W. Mück, Eur. Phys. J. C 73, 2679 (2013). arXiv:1310.6909 [hepth]

3. R. Casadio, A. Giugno, A. Giusti, Phys. Lett. B 763, 337 (2016). arXiv: 1606.04744 [hep-th]

4. R. Casadio, M. Lenzi, O. Micu, Phys. Rev. D 98, 104016 (2018). arXiv: 1806.07639 [gr-qc]

5. R. Casadio, M. Lenzi, O. Micu, Eur. Phys. J. C 79, 894 (2019). arXiv: 1904.06752 [gr-qc]

6. R. Casadio, A. Giugno, A. Giusti, M. Lenzi, Phys. Rev. D 96, 044010 (2017). arXiv: 1702.05918 [gr-qc]

7. R. Casadio, M. Lenzi and A. Ciarfella, Quantum black holes in bootstrapped Newtonian gravity. arXiv:2002.00221 [gr-qc]

8. G.A. Vilkovisky, Nucl. Phys. B 234, 125 (1984)

9. P. Ellicott, D.J. Toms, Nucl. Phys. B 312, 700 (1989)

10. D.V. Vassilevich, Phys. Rep. 388, $279 \quad$ (2003). arXiv:hep-th/0306138

11. A.O. Barvinsky, G.A. Vilkovisky, Phys. Rep. 119, 1 (1985)

12. A.O. Barvinsky, G.A. Vilkovisky, Nucl. Phys. B 282, 163 (1987)

13. A.O. Barvinsky, G.A. Vilkovisky, Nucl. Phys. B 333, 471 (1990)

14. J.S. Schwinger, J. Math. Phys. 2, 407 (1961)

15. L.V. Keldysh, Zh Eksp, Teor. Fiz. 47, 1515 (1964)

16. L.V. Keldysh, Sov. Phys. JETP 20, 1018 (1965)

17. X. Calmet, R. Casadio, F. Kuipers, Phys. Rev. D 100, 086010 (2019). arXiv:1909.13277 [hep-th] 